\title{
MODELAGEM DE DISTRIBUIÇÃO POTENCIAL DA ESPÉCIE Balfourodendron riedelianum (ENGL.) ENGL. (PAU MARFIM)
}

Lukas Rodrigues Souza - lukas@ufpr.br Universidade Federal do Paraná

Thomas Jeferson Vieira - thomas.jeferson.vieira@ hotmail.com

Universidade Federal do Paraná

Brenno Rodrigues Souza - brenno@alunos.utfpr.edu.br

Universidade Tecnológica Federal do Paraná 


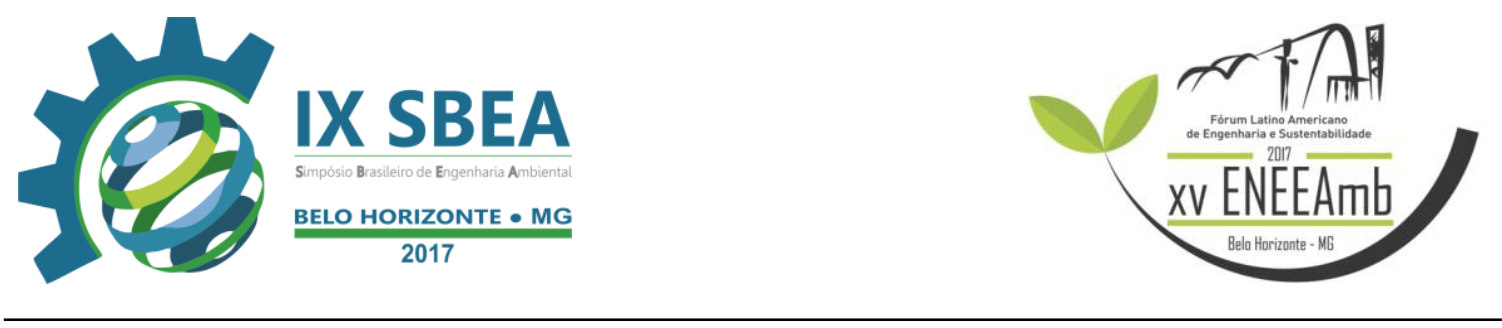

\section{RESUMO}

As alterações nos habitats naturais pelas ações antrópicas são uma das principais ameaças as comunidades vegetais. A qual a espécie B. riedelianum (pau marfim), pertencente ao Bioma Mata Atlântica, é considerada rara e ameaçada de extinção. Compreender de que maneira as alterações na paisagem influenciam as comunidades vegetais é de suma importância para o planejamento de áreas destinadas a preservação. Neste contexto o objetivo do presente trabalho consistiu em elaborar um modelo de distribuição geográfica potencial, através do algoritmo de entropia máxima, calculado pelo programa MAXENT 3.3, que utiliza dados de presença, dados climáticos e variáveis ambientais para estimar a distribuição geográfica potencial da espécie em questão. Gerou-se um mapa representativo de áreas com condições ambientais favoráveis ao desenvolvimento da espécie. Foi possível identificar áreas que originalmente eram ocupadas pelo Bioma Mata Atlântica, e que foram drasticamente reduzidas, em que a espécie $B$. riedelianum já foi registrada por diversos pesquisadores, que alertam pela necessidade de ações prioritárias em preservação, manejo, recuperação e implementação de novas Unidades de Conservação, gerando um Sistema de Unidades de Conservação nos Estados do Paraná, São Paulo, Santa Catarina, Rio Grande do Sul, Espirito Santo e Mato Grosso do Sul.

Palavras-chave: Modelagem de espécies vegetais, Balfourodendron riedelianum, Unidades de Conservação.

\section{INTRODUÇÃO/OBJETIVO}

Atualmente a destruição e a fragmentação de habitats pelas ações antrópicas são umas das principais ameaças a biodiversidade em populações e comunidades vegetais. Capazes de impactar negativamente na dinâmica e nas interações de diversas espécies, que sofrem com a alteração de suas taxas de desenvolvimento, de estrutura demográfica e de sucesso reprodutivo em remanescentes florestais (NASCIMENTO, 2007).

Neste contexto, a América Latina é um elemento importante, pois representa $12 \%$ de toda a superfície terrestre e contém dois terços de todas as espécies vegetais do planeta (BARBIERI, 2012). Especificamente no Estado do Paraná, os processos de exploração madeireira, seguidos da mineração, agricultura e pecuária, sem as devidas 


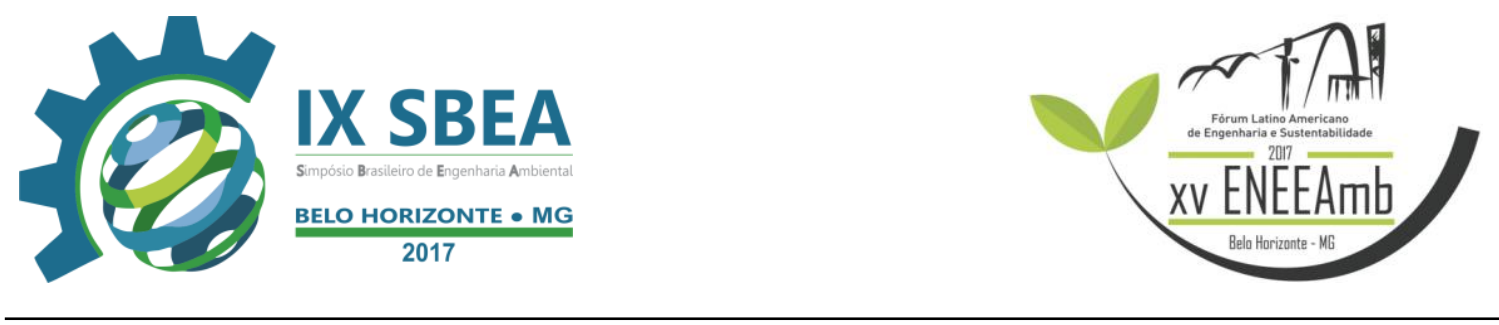

técnicas de manejo, reduziram drasticamente as populações e comunidades de diversas espécies vegetais nativas (RAMOS et al., 2010).

Dentre elas, de acordo com a Lista oficial de espécies ameaçadas de extinção do Instituto Ambiental do Paraná (IAP) a espécie B. riedelianum (pau marfim) pertencente à família Rutaceae, encontra-se ameaçada de extinção. Sendo que é considerada como rara por sua alta especialização em relação a seus habitats e nichos, em que a redução de suas áreas nativas preservadas e a falta de qualidade de habitats favoráveis afeta diretamente a sua capacidade de desenvolvimento, reprodução e dispersão (RAMBALDI \& DE OLIVEIRA, 2003).

Logo, compreender de que maneira as alterações na paisagem influenciam as comunidades vegetais é de suma importância, pois as áreas atuais destinadas a preservação e conservação são insignificantes e insuficientes para garantir a integridade da dinâmica populacional da maioria das espécies. Desta maneira ferramentas estratégicas para a conservação demandam por informações disponíveis para a biodiversidade, especialmente as que se referem à distribuição das espécies e seus nichos ecológicos.

Porém atualmente os dados fitogeográficos e os seus padrões de diversidade biológica são escassos (PIETCZAK, 2013). E a escolha sobre a destinação de uma área representativa para a diversidade biológica, levando em conta os ecossistemas, comunidades, populações, espécies e suas interações, com o objetivo principal de garantir a integridade das mesmas deve ser realizada a partir de critérios bem estabelecidos.

Em que se identifiquem os níveis de organização biológica mais importantes para a conservação e preservação, e os relacionem com as distribuições geográficas e as distribuições das unidades de conservação (MARGULES \& PRESSEY, 2000). Desta forma possibilita-se a identificação de regiões prioritárias para a conservação da espécie de interesse, levando em consideração seu padrão espacial de distribuição e suas interações (SCOTT et al., 1993). Nos quais os modelos de distribuição geográfica potencial permitem, através de metodologia empírica, a realização de inferências estatísticas sobre os possíveis processos ecológicos que influenciem a distribuição de organismos vegetais, estimando a distribuição geográfica potencial das mesmas 


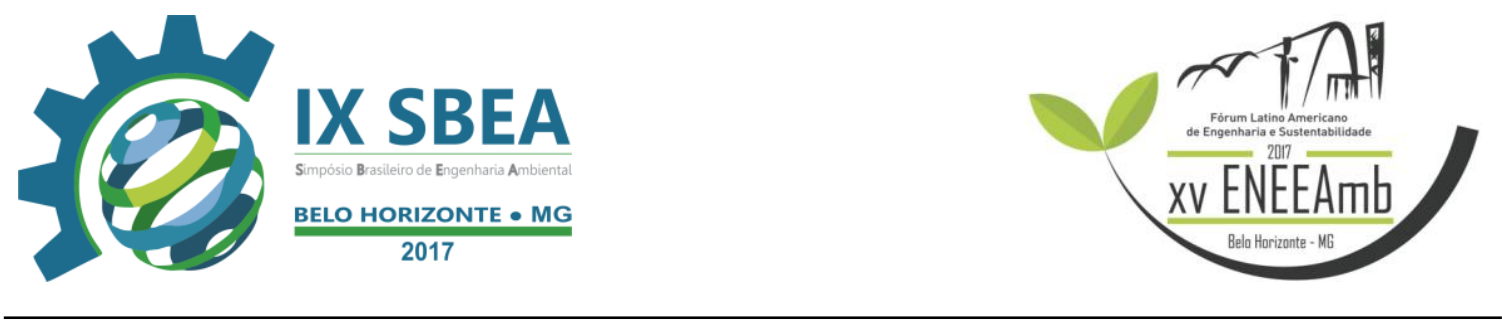

(MARGULES \& PRESSEY, 2000). E que podem ser utilizados como um elemento importante para o planejamento da conservação, e na identificação de áreas onde os esforços de conservação são mais necessários (SIMIÃO-FERREIRA \& DEMARCO JR, 2007).

Como resultado tem-se uma aproximação do potencial de locais onde as condições ambientais permitem a sobrevivência da espécie de acordo com as variáveis utilizadas, sem apontar a distribuição final da espécie devido a desconsiderar fatores evolutivos e históricos (PHILLIPS et al., 2006). Portanto objetiva-se modelar o potencial de distribuição atual da espécie $B$. riedelianum por meio da análise de dados bióticos, dados abióticos e a utilização de algoritmos como base de informação para estabelecimento de áreas prioritárias para a conservação e preservação.

\section{METODOLOGIA}

Criou-se um banco de dados com os pontos de ocorrência e as respectivas coordenadas geográficas da espécie $B$. riedelianum (pau marfim). Levantadas através de consultas ao banco de informações digitais da Lista de espécies da Flora do Brasil (http://floradobrasil.jbrj.gov.br) onde existem registros provenientes de coleções científicas de instituições e herbários do Brasil. Foram utilizados 64 pontos como registros de presença da espécie.

Para elaboração do modelo de distribuição potencial da espécie, foram utilizadas camadas que representam dados climáticos e variáveis ambientais. Todas essas providas pelo Intergovernmental Panel on Climate Change.

O modelo foi gerado a partir do programa MAXENT 3.3, que utiliza o algoritmo de entropia máxima, no qual considera como entrada os dados de presença e utiliza a área de estudo parcialmente ou completamente como contraponto. Esta técnica surgiu a partir da mecânica estatística por (PHILLIPS et al., 2004) e permite a modelagem da distribuição geográfica potencial de espécies. Apresenta a restrição de que o valor provável de cada variável ambiental da distribuição estimada corresponde a sua média empírica (ELITH \& LEATHWICK, 2006), porém apresenta a grande vantagem de sistematizar uma distribuição de probabilidades. 


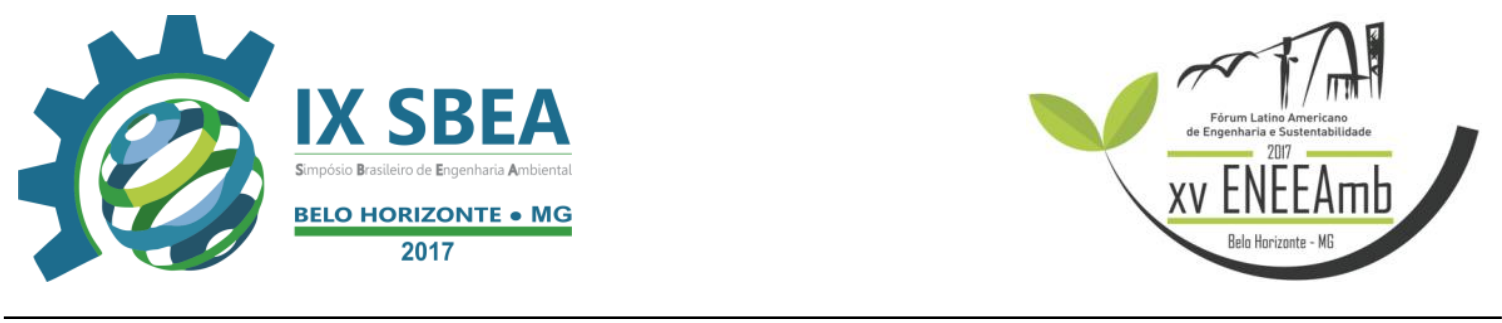

Sendo que os demais algoritmos produzem uma probabilidade para cada ponto. E o resultado apresentado pelo algoritmo é capaz de identificar outras áreas com características ambientais proporcionalmente similares aos pontos de ocorrência da espécie em uma escala de 0 a 100\%. A medida de entropia de Shannon (SHANNON, 1948) é dada por:

$$
S(p)=-\sum_{i=1}^{n} p_{i} \ln \left(p_{i}\right)
$$

Onde S(p) - entropia máxima da variável aleatória p; i - constante de ajuste dimensional, $\mathbf{n}$ - número total de categorias ou estados, $\boldsymbol{p}_{\boldsymbol{i}}$ - distribuição de probabilidade.

Gerou-se o modelo a partir dos registros de presença da espécie e as variáveis ambientais selecionadas inicialmente. Em seguida fez-se uso da ferramenta jackknife, teste do MAXENT 3.3, para analisar quais variáveis apresentaram uma maior contribuição para o modelo. Após essa análise realizou-se a regularização do modelo, com intuito de "suavizá-lo", torna-lo mais regular, retirando variáveis de pouca ou nenhuma contribuição, como sugerido por (ELITH \& LEATHWICK, 2006).

Como parâmetro de desempenho do modelo, foi utilizada a área sob a curva ROC (AUC), gerada a partir de dados independentes pelo próprio MAXENT 3.3. Metz (1986) relata que valores AUC entre 1,0 e 0,9 indicam modelos com excelente capacidade de previsão, entre 0,9 e 0,8 modelos com boa capacidade de previsão, entre 08 e 0,7 modelos capacidade de previsão média, e modelos com valor ACU inferior a 0,7 , indicam capacidade ruim de previsão.

\section{RESULTADOS E DISCUSSÃO}

Após a primeira execução do modelo, através da ferramenta jackknife no MAXENT 3.3, observou que algumas das 14 variáveis utilizadas inicialmente não apresentaram um ganho significativo para previsão de distribuição da espécie. Sendo necessário fazer a regularização do modelo, para assim obter-se maior precisão na predição de distribuição da espécie. Resultando em um mapa (Figura 1), onde as regiões 


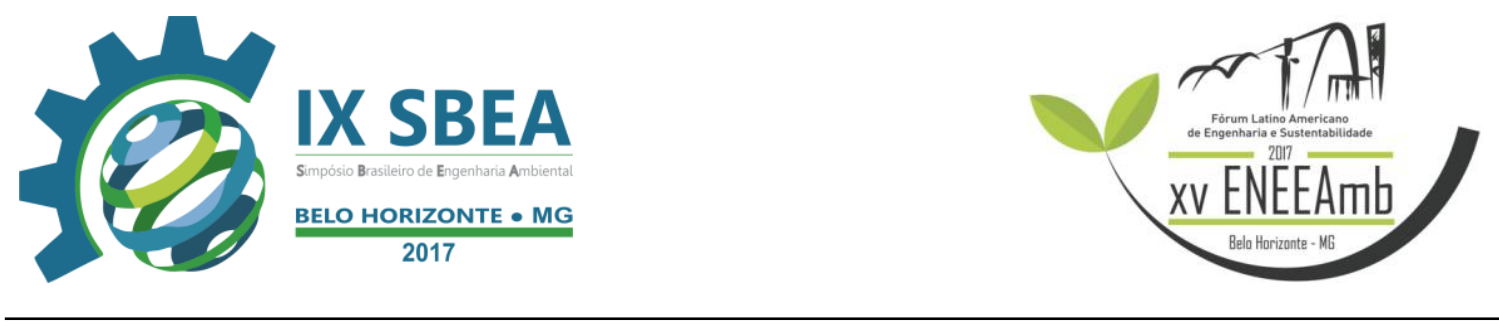

com cores mais quentes representam áreas com condições ambientais propícias para a espécie se desenvolver, enquanto que regiões em cores frias indicam áreas em que não há condições apropriadas para o desenvolvimento da espécie. Os pontos brancos no mapa indicam a localização dos pontos de registros de presença da espécie, utilizados como dados de entrada no modelo.

De acordo com o resultado do teste jackknife, a variável com ganho mais elevado foi a pressão de vapor, a qual, portanto, parece possuir maior quantidade de informação útil. A variável ambiental que diminui o ganho em maior proporção quando omitida é a precipitação em julho, a qual, portanto, parece possuir a maior quantidade de informação não presente em outras variáveis.

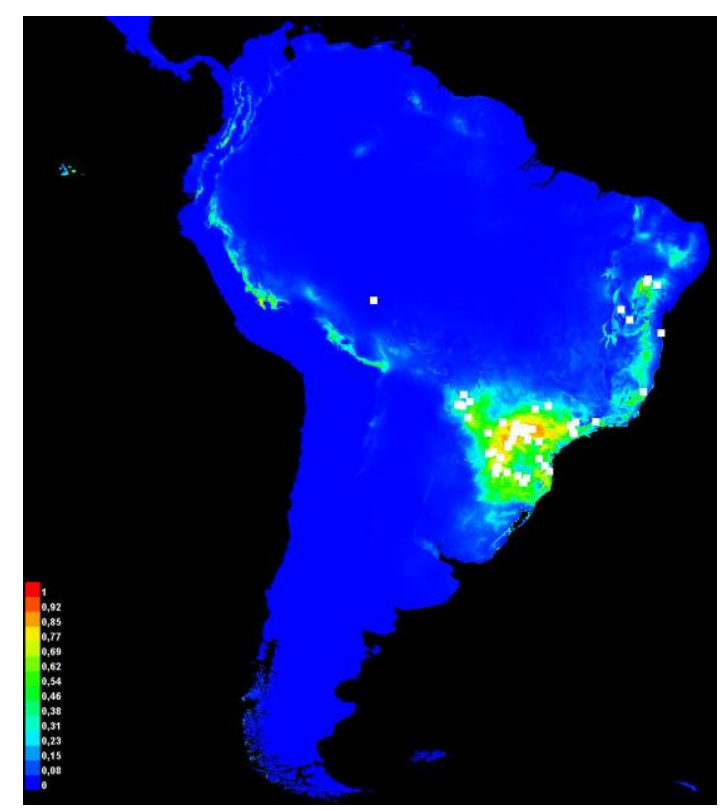

Figura 1 - Mapa representando potencial distribuição da espécie Balfourodendron riedelianum na América do Sul, gerado através do software MAXENT 3.3

A área sob a curva (AUC) resultou em um valor elevado. A especificidade é definida usando a área prevista, invés da comissão real, implicando que o valor máximo atingível de AUC é menor que 1. Porém em prática o teste AUC pode exceder esse limite. A partir dos resultados da modelagem potencial da espécie B. riedelianum, é possível evidenciar através dos pontos de registro da espécie (pontos brancos) que a 


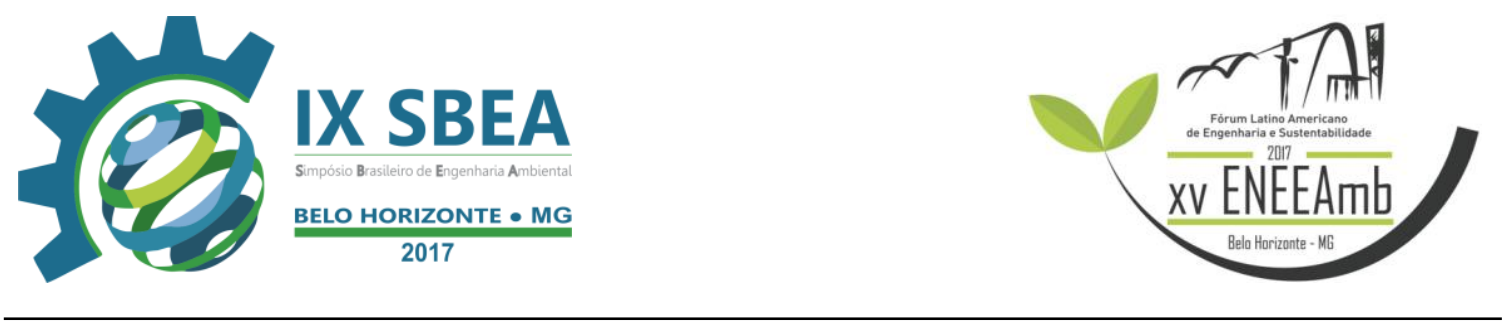

mesma ocorre preferencialmente nas regiões Sul, Suldeste e Centro-oeste, em vegetação florestal secundária e em ampla faixa altitudinal (CARVALHO, 2004).

B. riedelianum apresenta características biológicas como facilidade de dispersão e boa capacidade de regeneração natural, por ser anemocórica. Seus vetores de polinização são pequenos insetos (MORELLATO, 1991) e estimativas de densidade desta espécie apresentam em média 9 indivíduos por hectare com DAP maior ou igual a 5 cm (BORGHI, 2004; BIANCHINI, 2003; DURIGAN, 2000). Sendo pertencente ao grupo sucessional das secundárias tardias (DURIGAN \& NOGUEIRA, 1990). A falta de informações sobre suas populações torna o trabalho de suma importância para a garantia da integridade da espécie, a partir da previsão de locais onde se deve investir em programas de recuperação ambiental e delimitação de unidades de conservação integral.

As áreas estimadas pela modelagem de distribuição potencial como de maior adequabilidade ambiental para ocorrência da espécie envolvem ecossistemas associados ao bioma Mata Atlântica nos Estados do Paraná, São Paulo, Santa Catarina, Rio Grande do Sul, Espirito Santo e Mato Grosso do Sul. Estudos indicam que a espécie possui hábitos restritos a estes ecossistemas e as predições feitas pelo modelo podem ser consideradas como positivas (BERTANI et al., 2001; BORGHI, 2004; BIANCHINI, 2003; DURIGAN, 2000; RUSCHEL et al., 2009).

A distribuição prevista acompanha o que seria a área de cobertura original do bioma associado à espécie, com alto grau de necessidade de ações de preservação, conservação, recuperação e manejo pelos Estados pertencentes a estes territórios. Pois apenas $24 \%$ são áreas de proteção integral e cerca de $70 \%$ são pequenas demais $\left(<100 \mathrm{~km}^{2}\right)$ para garantir a persistência das espécies a longo prazo (SILVA \& TABARELLI, 2000). Que devem ser somadas de ações de conservação ex situ como a produção de sementes e auxílio na recomposição da espécie em áreas ambientalmente preferenciais para sua ocupação.

Pois os ecossistemas Floresta Estacional Semidecidual, Floresta Estacional Decidual e Floresta Ombrófila Mista são as regiões fitoecológicas da espécie em questão (CARVALHO, 2004). E que historicamente sofreram com a ocupação territorial em razão da industrialização e desenvolvimento econômico baseados na 
exploração irracional dos recursos naturais, com a consequente degradação do meio ambiente natural e da biodiversidade (TABARELLI et al., 2005).

O que evidencia a preocupante situação da espécie, que tem características ecológicas de ocupação relativamente agressivas e mesmo assim não é capaz de garantir a dinâmica de suas populações pelas interferências antrópicas, pela invasão de espécies exóticas invasoras e a perda de habitats (ANGELI \& MÜLLER, 2005). Em nível internacional a espécie em questão é considerada como em perigo (Lista Vermelha IUCN, 2011).

E faz com que a demanda por ações de preservação e conservação de modo sistêmico por todo o território dos Estados já citados sejam prioritários para garantir a integridade da biodiversidade, e consequentemente, da espécie em questão. Em que as Unidades de Conservação sejam projetadas adequadamente para otimizar a conservação in situ da biodiversidade, e garantir a variabilidade genética das populações e comunidades.

Além de que novas áreas de proteção sejam estabelecidas, para assim, ampliar as áreas e a expressividade das Unidades de Conservação Brasileiras (MITTERMEIER, 2005). Portanto o modelo de distribuição potencial da espécie foi capaz de identificar áreas que originalmente eram ocupadas pelo Bioma Mata Atlântica, e que foram drasticamente reduzidas, onde restam apenas $7 \%$ de sua cobertura original, representando menos de $100.000 \mathrm{~km}^{2}$ de área nativa (TABARELLI et al., 2005).

Onde a espécie $B$. riedelianum já foi registrada por diversos pesquisadores, que alertam pela necessidade de ações prioritárias em conservação e manejo de suas ilhas de remanescentes florestais, exíguas e distantes entre si. Que devido ao valor de sua madeira necessita de medidas de controle de exploração aliada a projetos de recuperação de suas populações naturais e estabelecimento de áreas de proteção integral (MESSINA, 2012), pois atualmente apenas 1,55\% dos remanescentes representam áreas protegidas (RODERJAN, 2002).

\section{CONCLUSÕES/RECOMENDAÇÕES}

A distribuição geográfica potencial de B. riedelianum compreende as áreas que originalmente representavam o Bioma Mata Atlântica e que pela supressão da vegetação 


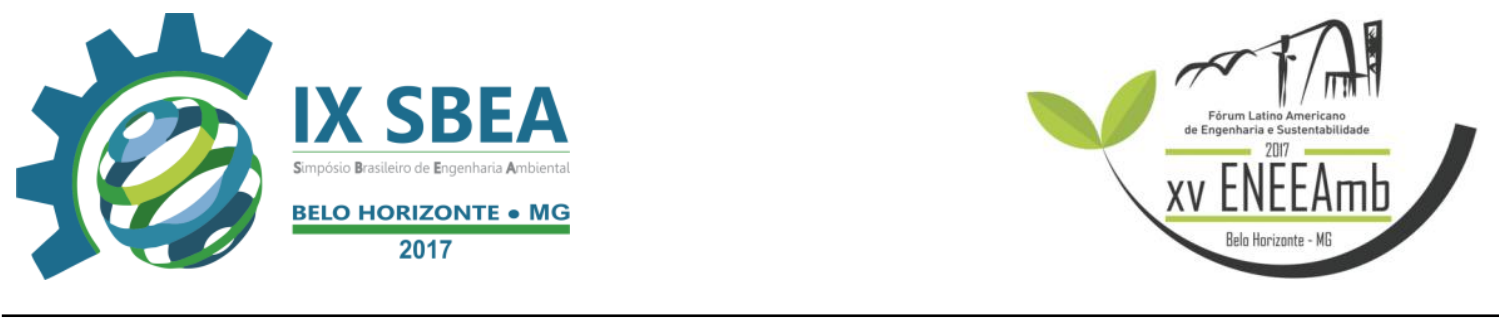

nativa para a agricultura, pecuária e silvicultura de modo extensivo foi reduzida drasticamente, onde restaram apenas fragmentos de vegetação de tamanhos insignificantes e distantes.

As Unidades de Conservação que abrangem os ecossistemas associados à espécie $B$. riedelianum não são representativas e não garantem a integridade da dinâmica das espécies vegetais e da biodiversidade local.

Sugere-se ações no que tange a implementação de novas Unidades de Conservação e medidas de Recuperação de Áreas Degradadas, gerando um Sistema de Unidades de Conservação. De modo que estas ações mitigadoras beneficiem a espécie em questão e todos os demais elementos de fauna e flora do Bioma em questão.

\section{REFERÊNCIAS BIBLIOGRÁFICAS}

ANGELI, A., MÜLLER, P. H. Balfourodendron riedelianum (Pau Marfim). Piracicaba: Ipef, 2005. 11p.

BARBIERI, E. A redução da biodiversidade. Cananéia: Núcleo de Pesquisa e Desenvolvimento do Litoral Sul, 2012. 16 p.

BERTANI, D. F.; RODRIGUES, R. R.; LUIZ, J. F. B.; SHEPHERD, G. J. Análise temporal da heterogeneidade florística e estrutural em uma floresta ribeirinha. Revista Brasileira de Botânica, v. 24, n. 1, p. 11-23, 2001.

BIANCHINI, E.; POPOLO, R. S.; DIAS, M. C.; PIMENTA, J. A. Diversidade e Estrutura de espécies arbóreas em área alagável do município de Londrina, Sul do Brasil. Acta Botanica Brasilica, v. 17, n. 3, p. 405-419, 2003.

BORGHI, W. A.; MARTINS, S. S.; QUIQUI, E. M. D.; NANNI, M. R. Caracterização e avaliação da mata ciliar à montante da Hidrelétrica de Rosana, na Estação Ecológica do Caiuá, Diamante do Norte, PR. Cad. Biodivers., v. 4, n. 2, p. 9-18, 2004.

CARVALHO, P. E. R. Pau-Marfim - Balfourodendron riedelianum. 93. ed. Colombo: Embrapa, 2004. 11 p.

DURIGAN, G.; FRANCO, G. A. D. C.; SAITO, M.; BAITELLO, J. B. Estrutura e diversidade do componente arbóreo da floresta na Estação Ecológica dos Caetetus, Gália, SP. Revista Brasileira de Botânica, v. 23, n. 4, p. 371-383, 2000.

DURIGAN, G.; NOGUEIRA, J. C. B. Recomposição de matas ciliares. São Paulo: Instituto Florestal, 1990. 14p. 


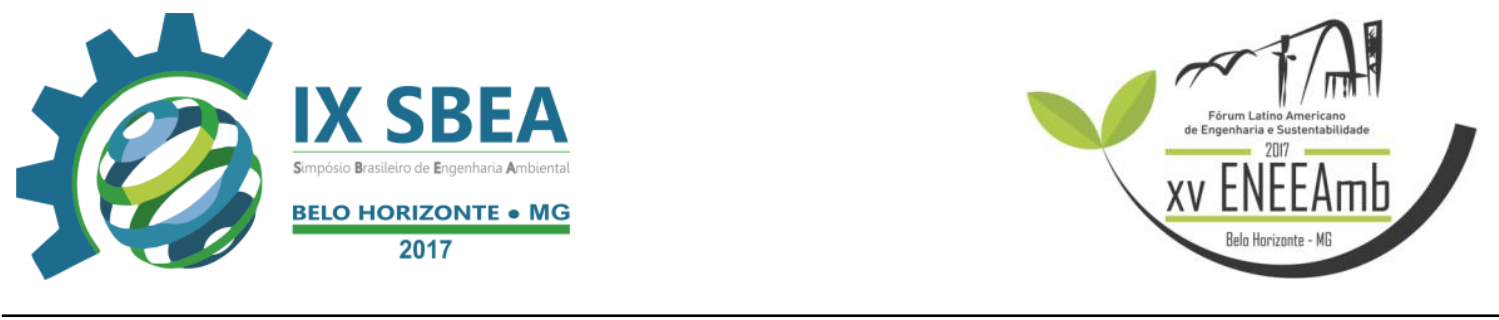

ELITH, J.; LEATHWICK, J. R. Species distribution models: ecological explanation and prediction across space and time. Annual Review Of Ecology: Evolution and Systematics, v. 40, p.677-697, 2006.

MARGULES, C. R.; PRESSEY, R. L. Systematic conservation planning. Nature, v. 405, n. 6783, p.243-253, 2000.

MESSINA, T. Avaliação de risco de extinção - Balfourodendron riedelianum (Engl.) Engl. Rio de Janeiro: CNC Flora, 2012. 5 p.

METZ, C.E. ROC methodology in radiologic imaging. Investigating Radiology, 21: 720-733, 1986.

MITTERMEIER, R. A. Uma breve história da conservação da biodiversidade no Brasil. Megadiversidade, v. 1, n. 1, p.15-21, 2005.

MORELLATO, L. P. C. Estudo da fenologia de árvores, arbustos e lianas de uma floresta semi-decídua no sudeste do Brasil. Campinas, 176 f., 1991. Tese (Doutorado) - Curso de Ecologia Florestal, Universidade Estadual de Campinas.

NASCIMENTO, S. M. do. Efeitos da fragmentação de habitats em populações vegetais. Campinas, 12 f., 2007. Dissertação (Mestrado) - Curso de Pós-graduação em Ecologia, Universidade Estadual de Campinas.

PHILLIPS, S. J.; ANDERSON, R. P.; SCHAPIRE, R. E. Maximum entropy modeling of species geographic distributions. Ecological Modelling, London, v. 190, n. 3-4, p.231-259, 2006.

PHILLIPS, S. J.; DUDIK, M.; SCHAPIRE, R. E. A maximum entropy approach to species distribution modeling. In Proceedings of the Twenty-first International Conference on Machine Learning. 2004. p.655-662.

PIETCZAK, C. Modelagem ecológica como ferramenta para a identificação de áreas prioritárias à conservação de Philodryas agassizii (SQUAMATA, DIPSADIDAE) no Rio Grande do Sul. Santa Maria, 55 f., 2013. Dissertação (Mestrado) - Curso de Programa de Pós-graduação em Biodiversidade Animal, Universidade Federal de Santa Maria.

RAMBALDI, D. M.; DE OLIVEIRA, D. A. S. Fragmentação de Ecossistemas: Causas, efeitos sobre a biodiversidade e recomendações de políticas públicas. 6 . ed. Brasília: Ministério do Meio Ambiente, 2003. 510 p.

RAMOS, E. A. DE P., IVANCHECHEN, E. M., PINHEIRO, E. S., MAXIMIANO, G. A., MARQUES, L. C., CASTELLA, P. R., SABÓIA, R. F., MASCARENHAS, T. B., MARQUES, T. P. Floresta Com Araucária - Volume 4. Curitiba, 2010. 


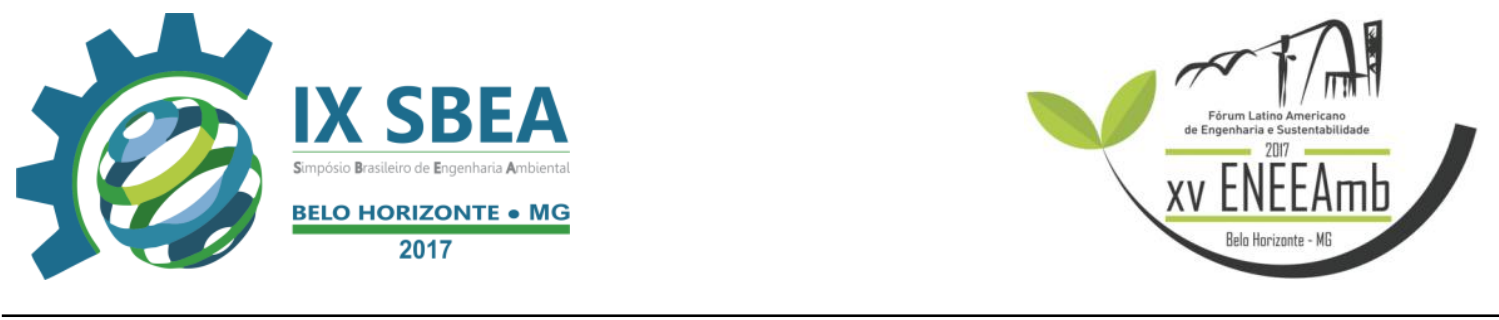

RODERJAN, C. V. As unidades fitogeográficas do Estado do Paraná, Brasil. Ciência e Ambiente, Curitiba, v. 24, n. 1, p.42-75, 2002.

RUSCHEL, A. R.; GUERRA, M. P.; NODARI, R. O. Estrutura e composição florística de dois fragmentos da floresta estacional decidual do Alto-Uruguai, SC. Ciência Florestal, v. 19, n. 2, p. 225-236, 2009

SCOTT, J. M. DAVIS, F.; CSUTI, B.; NOSS, R.; BUTTERFIELD, B.; GROVES, C.; ANDERSON, H.; CAICCO, S.; D'ERCHIA, F.; EDWARDS,T. C.; ULLIMAN, J. JR.; WRIGHT, R. Gap Analysis: A geographic approach to protection of biological diversity. Wildlife Monographs, n. 123, p.3-41, 1993.

SHANNON, C. E. A mathematical theory of communication. Bell Syst. Tech. J. 27. 379-423, 1948.

SILVA, J. M. C.; TABARELLI, M. Tree species impoverishment and the future flora of the Atlantic forest of northeast Brazil. Nature, 404: 72-74, 2000.

SIMIÃO-FERREIRA, J.; DEMARCO JR, P. Modelagem da distribuição potencial das espécies Tupiperla gracilis (Burmeister 1839) e Gripopteryx garbei (Navas 1936) (Gripopterygidae: Plecoptera) para a América do Sul. Anais do VIII Congresso de Ecologia do Brasil, Caxambu - MG. 2007.

TABARELLI, M.; PINTO, L. P.; SILVA, J. M. C.; HIROTA, M. M.; BEDÊ, L. C. Desafios e oportunidades para a conservação da biodiversidade na Mata Atlântica brasileira. Megadiversidade, v. 1, n. 1, p.133-137, 2005. 